\title{
Update on Diagnosing and Reporting Malignant Pleural Mesothelioma
}

\author{
Ivana Savic ${ }^{1}$, Jeffrey Myers ${ }^{2}$ \\ ${ }^{1}$ Institute of Pathology, Faculty of Medicine, University of Belgrade, Serbia, ${ }^{2}$ Department of Pathology, University of Michigan, \\ Ann Arbor, Michigan, United States
}

Correspondence: ivanasavic86@yahoo.com; Tel.: +3811136433409

Received: 6 March 2021; Accepted: 4 April 2021

\begin{abstract}
In this review, we summarize current approaches to diagnosis of malignant pleural mesothelioma, focusing on the distinction from benign mesothelial proliferations and other malignant tumors. Current recommendations for reporting histological subtype and tumor grade are also reviewed. Particular emphasis is placed on immunohistochemical and molecular tools that may help in establishing the diagnosis of mesothelioma with greater confidence. Immunohistochemical stains for BRCA1-associated protein (BAP1) and methylthioadenosine phosphorylase (MTAP) and homozygous deletion of p16 using fluorescence in situ hybridization (FISH) are emphasized as important methods for distinguishing benign from malignant mesothelial proliferations. Conclusions. Diffuse malignant pleural mesothelioma is a heterogeneous group of aggressive pleural tumors for which histological classification plays an increasingly important role in patient management. Stage and resectability remain key drivers of therapeutic strategies and outcomes. There is an increasingly robust suite of diagnostic tools, including immunohistochemical stains for BAP1 and MTAP and p16 FISH, for differentiating benign from malignant mesothelial proliferations in cytology and tissue specimens.
\end{abstract}

Key Words: Malignant Pleural Mesothelioma - Diagnostics • Immunohistochemistry • Fluorescence in Situ Hybridization.

\section{Introduction}

Malignant mesothelioma originates from mesothelial cells that line serosal surfaces (i.e., pleura, pericardium, peritoneum, tunica vaginalis). Pleura is the most frequently affected site, accounting for $70 \%$ to $80 \%$ of incident cases. Malignant pleural mesothelioma (MPM) is the most frequent primary malignant tumor of the pleura, and is characterized by aggressive behavior with mean survivals of 9 to 12 months.

Incidence and mortality from MPM is highly variable from one geographic region to the next, and is heavily influenced by the prevalence of mining and commercial applications of asbestos and the long latency periods between exposure and disease onset. In addition to occupational or household asbestos exposure, thoracic radiation in patients with breast carcinoma or Hodgkin lym- phoma has been identified as another environmental risk factor for MPM.

Mesothelioma most commonly affects individuals 60 years of age or older, with a male predominance. MPM is very rare in the pediatric population, with fewer than 300 cases reported in children. MPM commonly presents as an otherwise unexplained persistent pleural effusion. Features that tend to favor malignant over benign pleural disease include chest wall pain, hemorrhagic effusion, circumferential pleural thickening that includes involvement of mediastinal pleura, and nodular pleural thickening on computed tomography (CT) scans of the chest (1).

Malignant mesothelioma is a locally aggressive tumor that infiltrates the chest wall and lung parenchyma. Distant metastases are common in late stage disease. Autopsy studies demonstrate extrapleural metastases in almost $90 \%$ of individuals (2). Nodal 
metastases are a rare presenting manifestation of MPM and must be distinguished from benign nodal inclusions of mesothelial cells in patients with benign pleural or pericardial effusions (3).

The aim of this review article was to summarize the current approaches to diagnosis of malignant pleural mesothelioma, with a particular emphasis on its distinction from benign mesothelial proliferations and other malignant tumors using immunohistochemistry and molecular analyses.

\section{Cytology}

Cytological examination of pleural fluid is often the first, and sometimes the only, opportunity to identify patients with mesothelioma. Cytologic diagnosis alone is more limited than tissue biopsies in being able to establish specific histological subtypes or tumor grade, which may not significantly influence stage-dependent clinical management or prognosis for some patients. The sensitivity of cytology for MPM ranges from $30 \%$ to $75 \%$, meaning that a negative cytology does not exclude the diagnosis (4). Liquid based processing and/or cell blocks can improve diagnostic performance (5). The International Mesothelioma Interest Group clusters cytology results from patients with MPM into three categories: 1. Malignant based on cytomorphological criteria, 2. Malignant on the basis of supportive ancillary studies, and 3. Nondiagnostic (6). Cytological features of MPM include hypercellularity of specimen, presence of tissue fragments, enlarged mesothelial cells with enlarged nuclei resulting in high nuclear:cytoplasmic ratios, macronucleoli, papillary three dimensional spheres, and acidophilic extracellular matrix. A "cell in a cell" phenomenon and membrane protrusions or blebs have also been described. Malignant epithelioid mesothelial cells share some cytologic features with reactive, non-neoplastic mesothelial cells such as scalloped borders of cell groups and presence of intercellular windows exhibiting lighter, dense cytoplasm edges. Effusion specimens characterized by cytologically malignant cells often require immunohistochemical studies to confirm mesothelial origin (see Table
1). Ancillary studies, such as immunohistochemistry for BRCA1-associated protein (BAP1) and methylthioadenosine phosphorylase (MTAP), and p16 fluorescence in situ hybridization (FISH), are often required to establish a cytological diagnosis of MPM with greater confidence, and can substantially improve diagnostic sensitivity.

\section{Histology}

The 2015 WHO classification of pleural tumors divides diffuse malignant mesothelioma into three main histological subtypes: Epithelioid (60\%$80 \%)$, sarcomatoid $(<10 \%)$, and biphasic $(10 \%$ $15 \%)$. The relative frequency is higher for biphasic subtypes and lower for sarcomatoid subtypes in surgical specimens compared to pre-resection biopsies, indicating the importance of sampling in accurate classification of malignant pleural tumors (7). It is important to distinguish between these subtypes because of stage-dependent differences in therapeutic strategies and differences in average lengths of survival. Retrospective cohorts drawn from large multi-institutional data sets consistently show the longest overall survivals in patients with epithelioid histology, the shortest survivals in those with sarcomatoid MPM, and intermediate survival in patients with biphasic MPM (8). Truly localized mesotheliomas are extremely rare, show the same range of histologic subtypes, and are affiliated with better survivals given the possibility of complete surgical excision (9).

\section{Epithelioid Mesothelioma}

Epithelioid mesothelioma, the most common form of MPM, usually comprises mildly atypical low columnar to cuboidal cells resembling reactive mesothelial cells arranged in a variety of growth patterns including most commonly a tubulopapillary architecture (Figure 1). A recent proposal from a large international multidisciplinary group suggested subclassifying epithelioid mesothelioma by specific architectural patterns and, in some variants, unique cytological features (10). The most common architectural patterns include tubu- 


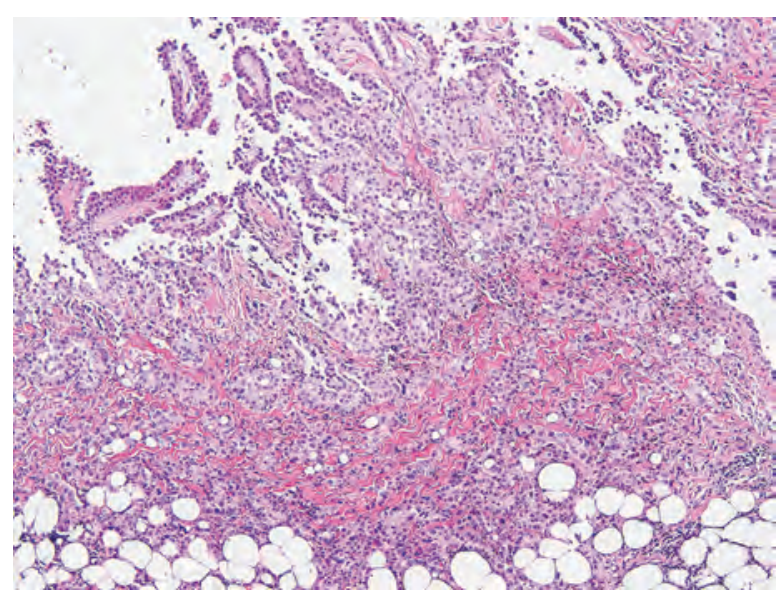

Figure 1. Epithelioid mesothelioma. Low magnification photomicrograph showing tubulopapillary mesothelioma composed of relatively bland non-mucinous cuboidal cells forming a combination of tubules (left center), papillae (upper left) and small irregularly shaped solid groups and cords (hematoxylin and eosin stain; original magnification 40x.

lopapillary, solid, and trabecular, while micropapillary, adenomatoid (microcystic), clear cell, transitional, deciduoid, small cell, and lymphohistiocytic variants are relatively rare. Pleomorphic subtypes are defined as epithelioid mesotheliomas in which more than $10 \%$ of tumor cells show marked nuclear pleomorphism; this subtype is associated with the worst overall survival (8.1 months) among epithelioid MPM (11). The international proposal includes recommendations for reporting percentages of the various architectural patterns and cell types for extrapleural pneumonectomy and extended pleurectomy/decortication surgical specimens, a reporting practice for which there is no evidence regarding its value and therefore not widely adopted outside of a research setting.

\section{Grading of Epithelioid Mesothelioma}

Histological subtype and TNM stage drive therapeutic strategies in patients with MPM. Several retrospective case series have demonstrated limited utility of histologic grading for epithelioid mesotheliomas, usually based on some combination of nuclear grade, mitotic rate, and necrosis. Grading of MPM has not yet been adopted in routine practice and is not included in current cancer reporting templates from the College of American Pathologists. In their previously referenced proposal, an international multidisciplinary group recommended a two-tier system of grading based on nuclear grade and necrosis. In this proposed system, low-grade MPM comprises nuclear grade 1 with or without necrosis and nuclear grade 2 without necrosis; high-grade is reserved for tumors with nuclear grade 2 and necrosis or nuclear grade 3 with or without necrosis (10). In making a recommendation for a practice not yet widely adopted the authors suggest that tumor grading may be of benefit in stratifying patients for clinical trials or adding greater precision to the risk stratification currently provided by histological subtyping. While this may eventually emerge as a standard reporting element, in our view it should be optional for pathology reporting given the absence of compelling evidence regarding its value outside of a research setting. It also should be emphasized that this proposed grading system was recommended only for epithelioid mesothelioma; other types of MM (sarcomatoid MM, and sarcomatoid parts in biphasic MM) are inherently more aggressive and therefore high grade by definition.

\section{Sarcomatoid Mesothelioma}

Sarcomatoid mesothelioma is less frequent but more aggressive than epithelioid mesothelioma, with mean survivals of 3.5-8 months (12). Differentiating between epithelioid and sarcomatoid $\mathrm{MM}$ is important because of stage-dependent differences in treatment approach (13). Sarcomatoid mesothelioma is characterized by neoplastic spindle cells exhibiting variable numbers of mitoses and degrees of cytologic atypia (Figure 2). The cells are typically arranged in vaguely fascicular growth patterns thus resembling soft tissue sarcomas ("sarcomatoid"). Histological subtypes of sarcomatoid MPM include conventional (44\%), desmoplastic (34\%), sarcomatoid with desmoplastic areas $(21 \%)$, sarcomatoid with heterologous elements (1\%), and lymphohistocytoid mesotheliomas $(<1 \%)(14)$. 


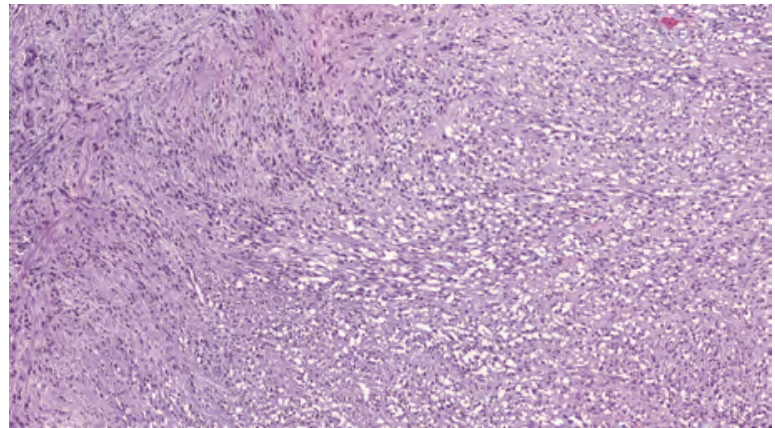

Figure 2. Sarcomatoid mesothelioma. Intermediate magnification photomicrograph showing neoplastic spindles cells arranged in a loosely organized fascicular growth pattern with invasion into chest wall soft tissue at upper left (hematoxylin and eosin stain; original magnification 132x).

Desmoplastic mesothelioma is a common subtype and is the most challenging to distinguish from benign fibrosing pleuritis, sometimes referred to as fibrous pleurisy. Desmoplastic MPM is paucicellular, with random variation in cellularity across a relatively narrow range. The areas showing an abrupt increase in cellularity comprise mildly atypical spindle cells with enlarged hyperchromatic nuclei arranged in a "patternless pattern of Stout" with abundant collagenous stroma (Figure 3). Keys to diagnosis are a combination of this distinctive storiform histology, invasion of chest wall soft tissue and/or lung parenchyma, bland necrosis characterized by dropout of basophilic nuclei, focal areas with frankly malignant sarcomatoid histology, and/or distant metastases (15). Immunohistochemical stains for cytokeratins are of limited value since non-neoplastic reactive spindled mesothelial cells are also positive, but can be helpful in identifying areas of chest wall invasion (16).

Transitional mesothelioma, traditionally considered a rare architectural and cytological subtype of epithelioid MPM, comprises cohesive plump spindle cells with elongated ambiguous cytomorphology. Recent studies indicate that transitional mesothelioma is genetically more closely related to sarcomatoid MPM, and recommend that it be considered a subgroup of sarcomatoid mesothelioma $(17,18)$.
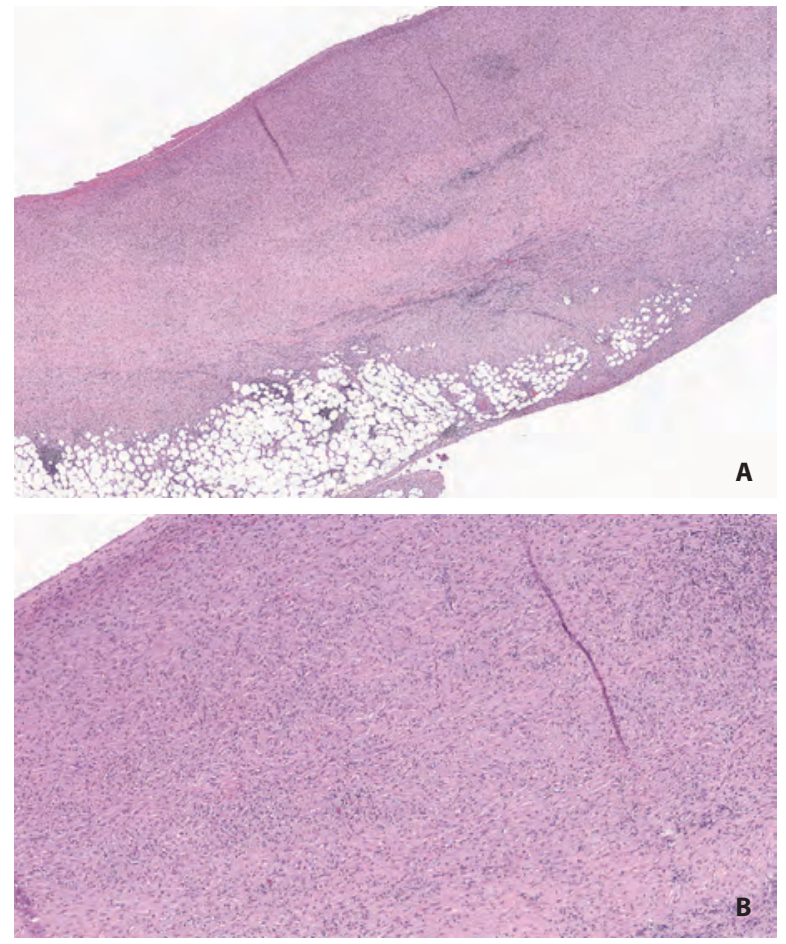

Figure 3. Desmoplastic mesothelioma. A) Low magnification photomicrograph showing thickened parietal pleura with random variation in cellularity and invasion of chest wall fat (hematoxylin and eosin stain; original magnification 27×). B) Intermediate magnification photomicrograph showing more cellular zone in which neoplastic spindle cells are arranged in the vaguely storiform "patternless pattern of Stout" (hematoxylin and eosin stain; original magnification 100x).

\section{Biphasic Mesothelioma}

Biphasic mesothelioma is defined as showing a combination of epithelioid and sarcomatoid histologies, with each component comprising more than $10 \%$ of the tumor (Figure 4). A sarcomatoid component of less than $80 \%$ in biphasic MM has been linked to improved survival. Interobserver agreement in diagnosis of biphasic mesothelioma is moderate (Kappa $=0.45$ ), suggesting that updating the definition of biphasic MPM is needed to support more consistent risk stratification (19). Although fibrous stroma in epithelioid MPM is typically scant, it is sometimes florid and thus can mimic biphasic MPM. Cases in which it is uncertain whether the sarcomatoid component represents a benign florid stromal reaction or a prolif- 


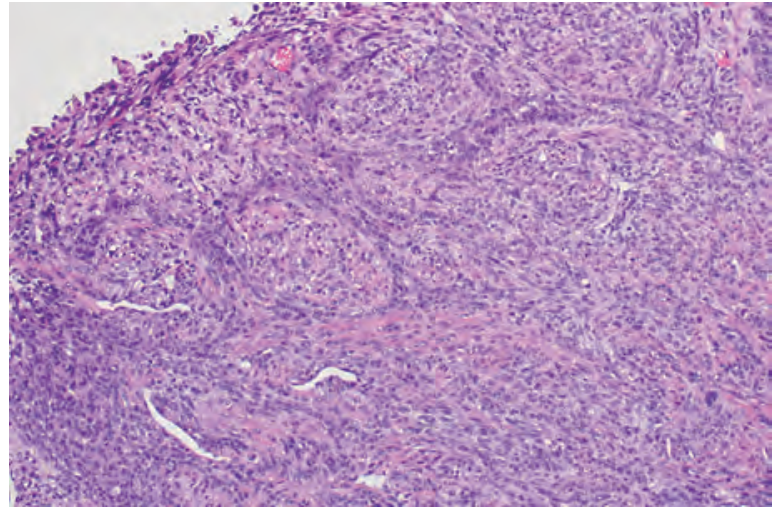

Figure 4. Biphasic mesothelioma. Photomicrograph showing a glandular epithelial component and a less differentiated stromal component comprising spindle cells (hematoxylin and eosin stain; original magnification 100x).

eration of neoplastic mesothelial cells may be resolved by demonstrating homozygous deletion of p16 using a FISH technique (20).

\section{Immunohistochemical Stains for Diagnosis}

Histopathological diagnosis of MPM begins with careful examination of routinely stained sections in an appropriate clinical and radiological context. Immunohistochemical stains can be extremely helpful in distinguishing MPM from other malignancies capable of diffuse pleural involvement that may mimic mesothelioma ("pseudomesotheliomatous"), and in separating MPM from benign mesothelial proliferations. Pancytokeratin stains may be useful in separating MPM from other non-epithelial mimics, such as metastatic melanoma or diffuse high-grade lymphomas confined to the pleura and pleural space. They should be interpreted with caution, given that reactive spindle cells of mesothelial origin are also keratin and calretinin positive (Figure 5). A small minority of sarcomatoid MPM may be keratin negative. Epithelioid MPM is typically positive for cytokeratin 7 as are many of the entities frequently considered in the differential diagnosis, which may limit its utility. Epithelioid MPMs are also frequently positive for high molecular weight cytokeratins using antibodies directed against cytokeratins 5 and 6; staining for high molecular cytokeratins is less common in sarcomatoid types (21).
It is important to first establish a working diagnosis based on routinely stained sections and knowledge of the clinical and radiological findings before deciding on immunohistochemical stains likely to be of value. Choice of immunohistochemical markers to distinguish MPM from other entities with epithelioid phenotypes depends heavily on the histologic subtype being considered (epithelioid or sarcomatoid), location of the neoplasm (pleura or peritoneum), and the types of tumors included in the differential diagnosis (e.g., squamous cell carcinoma, adenocarcinoma, epithelioid hemangioendothelioma, melanoma). Given that none of the markers have $100 \%$ specificity, a limited panel that includes antibodies with sensitivity or specificity of at least $80 \%$ is recommended (22). An immunohistochemical panel should contain at least two mesothelial markers and two markers appropriate to the working diagnoses established on the basis of routinely stained sections and any pertinent history including previously diagnosed malignancies. For confirmation of mesothelial origin in patients suspected of having epithelioid or biphasic MPM, calretinin, WT-1 (nuclear staining only), cytokeratin 5/6, and D2-40 (podoplanin) are useful markers (23). Markers useful for tumors in which metastatic carcinoma is a diagnostic possibility include MOC31, BG8, CEA, claudin 4, and BerEP4. MOC31 and BerEP4 target the same transmembrane glycoprotein (EpCAM), and therefore the final choice of markers should include one, rather than both of them. In addition to two general carcinoma markers, immunostains that are specific for certain carcinoma subtypes may be helpful. This is dependent not only on the histologic findings but also on relevant clinical and radiological information (i.e., previous malignancies or suspicion of other primary sites at presentation). In patients suspected of having metastatic adenocarcinoma for which no primary is known, TTF-1 may be helpful since lung is the most frequent source for metastatic adenocarcinomas with a pseudomesotheliomatous growth pattern (24). Major differential diagnoses and immunohistochemical markers useful for differentiating MPM from other malignant neoplasms are summarized 

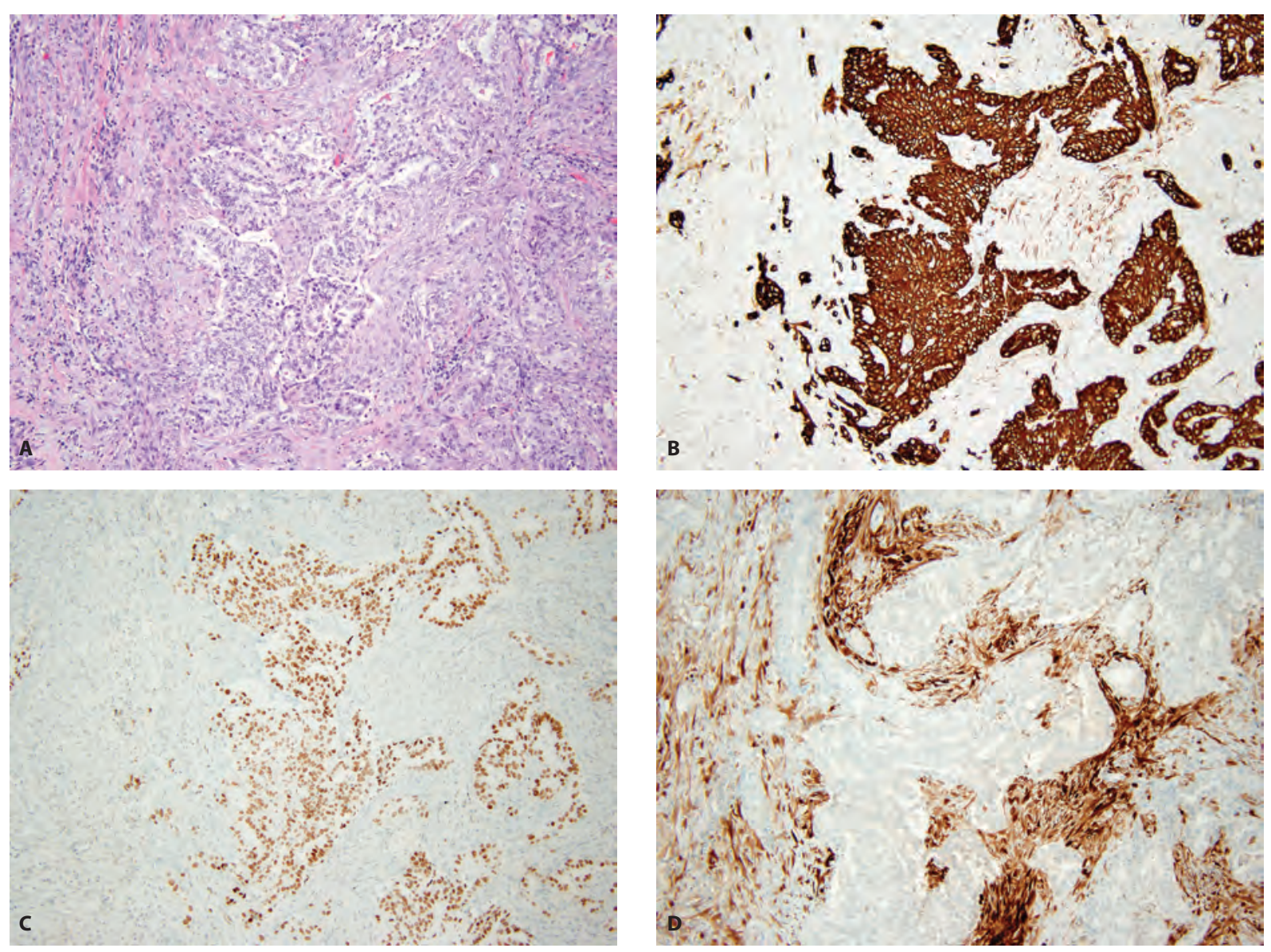

Figure 5. Metastatic adenocarcinoma of the lung. A) Photomicrograph showing glandular neoplasm infiltrating the parietal pleura with a variably cellular stromal response resembling biphasic MPM (hematoxylin and eosin stain; original magnification 100x). B-D) Photomicrographs showing immunostains performed on this pleural biopsy. Both the metastatic adenocarcinoma and the stromal cells, which include non-neoplastic spindle cells of mesothelial origin, are positive for cytokeratin 7 (B). Staining for TTF-1 (C) is limited to the adenocarcinoma, and calretinin (D) to non-neoplastic mesothelial cells (original magnification 100x).

in the Table 1. Immunohistochemistry for BAP1 is used primarily to separate benign from malignant mesothelial proliferations (see following section, Separating malignant from benign mesothelial proliferations), but can also be helpful in selected circumstances for distinguishing MPM from carcinomas in malignant pleural effusion cytology specimens, with high sensitivity $(87 \%)$ and specificity (98\%) (25).

Immunohistochemistry for sarcomatoid MPM often begins with cytokeratin stains to establish an epithelioid phenotype and exclude soft tissue sarcomas. In a large retrospective case series of over 300 cases, $93 \%$ of sarcomatoid mesotheliomas ex- pressed cytokeratins; sensitivity increased with use of an antibody cocktail (e.g., AE1/AE3 \pm CAM5.2), more extensive tumor sampling, and adequate tissue fixation (14). Keratin staining may be negative in the osteosarcomatous or chondrosarcomatous components of sarcomatoid MPM with heterologous elements (22). Immunohistochemical markers are less useful for distinguishing sarcomatoid MPM from sarcomatoid carcinomas. Calretinin is negative in $>50 \%$ of sarcomatoid mesotheliomas, as is nuclear staining for WT-1 (21). Strong diffuse staining for GATA-3 is nearly universal in sarcomatoid MPM and, along with radiological distribution of disease (i.e., localized versus diffuse), 
Table 1. Immunohistochemical Stains Useful for Separating MPM from Malignant Mimics

\begin{tabular}{|c|c|c|}
\hline Histologic MPM type & Mesothelial markers & $\begin{array}{l}\text { Markers more commonly expressed in } \\
\text { non-mesothelial neoplasms }\end{array}$ \\
\hline \multirow{6}{*}{ Epithelioid } & \multirow{6}{*}{$\begin{array}{l}\text { CK AE1/3 +, calretinin +, WT- } 1+\text {, } \\
\text { CK5/6 +, mesothelin +, D2-40 + }\end{array}$} & Lung adenocarcinoma (TTF-1 +, napsin A +) \\
\hline & & Adenocarcinoma, NOS (CEA+, claudin 4+, MOC31/Ber-EP4+, BG8+) \\
\hline & & Squamous cell lung carcinoma (p40 + MOC-31/Ber-EP4+) \\
\hline & & Renal cell carcinoma (PAX8 +, CAIX +) \\
\hline & & Breast carcinoma $(\mathrm{ER}+, \mathrm{PR}+, \mathrm{GCDFP}-15+$, mammaglobin + , GATA3 +) \\
\hline & & Epithelioid hemangioendothelioma (CD31 +, CD34 +, FLI-1 +, ERG +) \\
\hline \multirow{3}{*}{ Sarcomatoid } & \multirow{3}{*}{$\begin{array}{l}\text { CK AE1/3 +, CAM5.2 +, D2-40 +, } \\
\text { calretinin +, WT-1 +, GATA3 + }\end{array}$} & Sarcomatoid carcinoma (CK AE1/3 + CAM 5.2 +, GATA3 -). \\
\hline & & Angiosarcoma (CD31 +, CD34 +, ERG +) \\
\hline & & Synovial sarcoma (CD99 +, TLE-1 +) \\
\hline
\end{tabular}

MPM=Malignant pleural mesothelioma

can be especially helpful for this frequently challenging differential diagnosis (26).

\section{Separating Malignant from Benign Mesothelial Proliferations}

There are a number of histological features helpful in separating MPM from benign mesothelial proliferations. Invasion of chest wall soft tissues and/or pulmonary parenchyma is the single most helpful finding in establishing a diagnosis of MPM (Figure 6) $(1,16)$. Cytokeratin stains may be helpful in demonstrating invasion not otherwise easily observed with routinely stained sections alone. Reactive mesothelial cells do not invade the surrounding tissues, but "pseudo invasion" may occur when benign mesothelial cells are entrapped in the fibrosis characteristic of fibrosing pleuritis resulting in a distinctive pattern of layering resembling the annual growth rings in trees (Figure 7). Inflammation of the pleura with associated mesothelial hyperplasia tends to have a predictably zonal distribution of cellularity in which the cellularity is greatest adjacent to the pleural space and gradually or abruptly diminishes as it approaches the chest wall interface. Benign mesothelial proliferations may include papillary structures but they lack the complex stratification characteristic of MPM and instead comprise simple, non-arborizing structures lined by a single layer of cells. In addition, reactive proliferations often are accompanied by surface fibrin and granulation tissue in which capillary sized vascular spaces are arranged in parallel perpendicular to the pleural surface.

Loss of BAP1 expression and homozygous deletion of p16 have become diagnostic methods for separating benign from malignant mesothelial proliferations with greater frequency and accuracy, thus increasing diagnostic sensitivity for MM. A growing number of studies attest to their practical value in the diagnostic process (27).

BAP1 is a cellular enzyme with tumor suppressor functions. It is involved in cycle-cell progression, repairing ionizing radiation-induced DNA damage, regulation of gene expression and chromatin remodeling. Early studies showed lack of BAP1 immunoreactivity due to somatic $B A P 1$ genetic alterations, such as deletions or point mutations, in more than $40 \%$ of MPMs (28). More recent studies show loss of BAP1 expression in nearly $75 \%$ of epi-

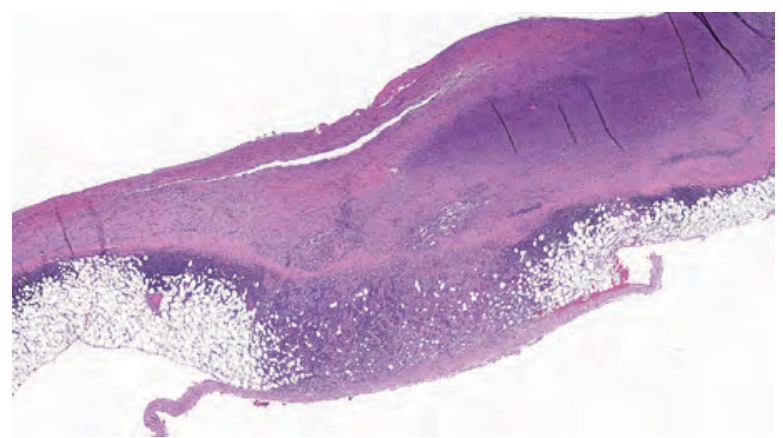

Figure 6. Epithelioid mesothelioma with chest wall invasion (hematoxylin and eosin stain; original magnification 19x). 


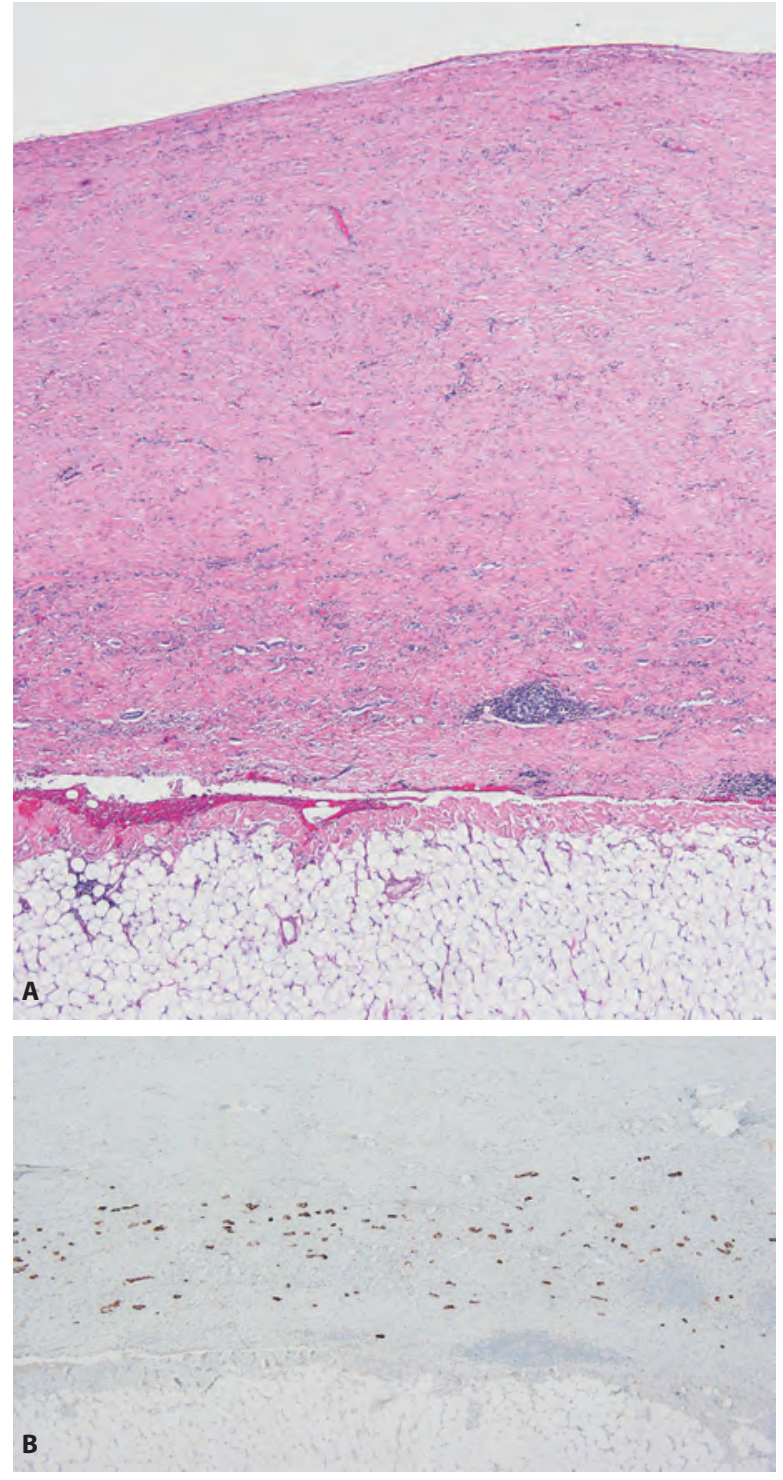

Figure 7. Fibrosing pleuritis ("fibrous pleurisy") with layering of entrapped mesothelial cells. A) Low magnification photomicrograph showing fibrous thickening of parietal pleura and a sharp interface with chest wall adipose tissue. Entrapped mesothelial cells are arranged in a linear fashion in the lower third without the random variation in cellularity and invasive growth patterns more characteristic of mesothelioma (hematoxylin and eosin stain; original magnification 20x). B) Photomicrograph of immunohistochemical stain for cytokeratins (AE1/AE3 and CAM5.2 cocktail) showing the layering of entrapped mesothelial cells and the absence of chest wall invasion (original magnification 40x).

thelioid MPM with lower sensitivities on the order of $50 \%$ for biphasic and $10 \%$ or less for sarcomatoid MPM. Loss of BAP1 expression has been as- sociated with younger age at onset and improved median survival in MPM, although BAP1 expression as a prognostic biomarker remains controversial (29). Loss of BAP1 expression is manifested as complete, and rarely partial, loss of nuclear staining in tumor cells with positive staining of internal controls (i.e., inflammatory cells and stromal cells) (Figure 8). BAP1 loss occurs in both sporadic and familial MPM, the latter linked to germline BAP1 mutations $(30,31)$. BAP1 loss has consistently shown $100 \%$ specificity for distinguishing malignant from benign mesothelial proliferations; benign reactive mesothelial proliferations always retain nuclear BAP1 expression. BAP1 expression is also retained in adenomatoid tumors, a lesion that only rarely occurs in the chest although adenomatoid tumor-like histology has been well described in MPM (32). In a comparison of two retrospective cohorts, Erber showed that BAP1 loss occurred only in mesotheliomas and was retained in all 42 genital adenomatoid tumors (33). BAP1 immunohistochemistry is a powerful addition to the growing portfolio of diagnostic tools for atypical mesothelial proliferations, but it is important to remember that the variable sensitivity of BAP1 loss in MPM limits its negative predictive value: retention of BAP1 expression by itself cannot be used to exclude a diagnosis of mesothelioma.

Loss of nuclear expression of 5-hydroxymethylcytosine $(5-\mathrm{hmC})$ has shown promise as an additional immunohistochemical stain for distinguishing malignant from benign mesothelial proliferations with high (92\%) sensitivity and 100\% specificity, although this has not yet been widely adopted (34). Several other markers, including desmin, epithelial membrane antigen (EMA), p53, IMP3, GLUT-1, CD146, and CD147, have shown only limited diagnostic value and are unlikely to be useful in individual cases (1).

Homozygous deletion of $9 \mathrm{p} 21$ is an important method for separating benign from malignant mesothelial proliferations. This region comprises genes for two cyclin-dependent inhibitor kinases, CDKNA2A (p16) and CDKN2B, and MTAP. CDK$N 2 A$ is present in normal cells where it is involved in cell cycle regulation. Deletion of p16 is present 


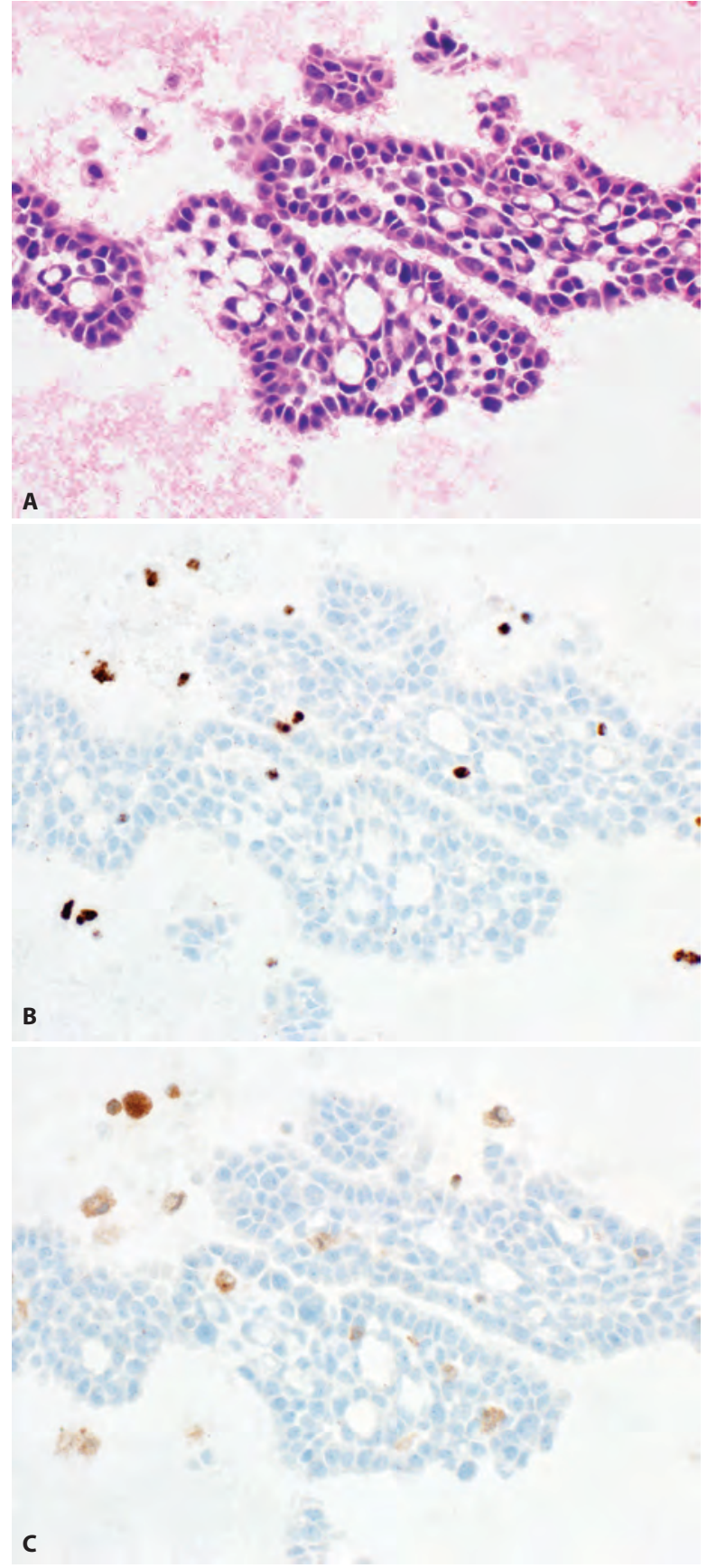

Figure 8. Immunohistochemical markers for separating mesothelioma from benign mesothelial proliferations. A) High magnification photomicrograph showing two-dimensional groups of atypical mesothelial cells in a cell block prepared from pleural fluid (hematoxylin and eosin; original magnification 400x). B-C) Immunohistochemical stains performed on the same cell block showed loss of nuclear staining for BAP1 (B) and loss of both cytoplasmic and nuclear staining for MTAP (C) with appropriately staining internal controls (original magnification 400x). in as many as $90 \%$ of sarcomatoid mesotheliomas, and about $70 \%$ of desmoplastic subtypes, but tends to be less common in epithelioid and biphasic mesotheliomas. Practically, $p 16$ deletion is useful for distinguishing benign mesothelial proliferations from MPM, but cannot reliably distinguish MPM from other carcinomas in which $p 16$ may also be deleted $(35,36)$.

Homozygous p16 deletion is demonstrated with a FISH technique using centromere 9 and CDKN2A probes, and can be applied to both cytology and histology specimens. Overall sensitivity of $p 16 \mathrm{FISH}$ in effusion cytology specimens is between $56 \%$ and $79 \%$ with a high $(100 \%)$ positive predictive value given $100 \%$ specificity. False negative $p 16$ FISH may occur due to admixed reactive mesothelial cells that may be morphologically indistinguishable from malignant mesothelial cells (22). In histology specimens, the sensitivity of $p 16$ FISH for epithelioid and biphasic MM ranges between $45 \%$ and $85 \%$. Homozygous p16 deletion has been associated with shorter overall survival.

$M T A P$, a tumor suppressor gene co-located with $C D K N 2 A$, is often deleted with $p 16$, making immunohistochemical staining for MTAP protein a reasonable surrogate for p16 FISH (37). Negative cytoplasmic staining for MTAP in tumor cells with positive cytoplasmic and nuclear staining in positive internal controls, such as inflammatory and stromal cells, favors the diagnosis of $\mathrm{MM}$ with $100 \%$ specificity and a sensitivity of around $45 \%$ in tissue sections and cytology cell blocks. Combination with BAP1 immunohistochemistry increases sensitivity to around $75 \%$ to $80 \%$ (37).

Other emerging methods that are used less frequently, in part because of technological challenges that serve as barriers to access, include hemizygous deletion of neurofibromatosis type 2 (NF2 gene) on 22q12 and gene expression arrays (38, 39). Bruno and colleagues showed that expression analysis of 117 genes using a nanoString System in a relatively small number of cases performed better than BAP1 and $p 16$ FISH, with overall sensitivity of $95.6 \%$ and $100 \%$ specificity (38). There are no currently recommended predictive biomarkers for patients with MPM, although that may change 
as research continues with the hope of identifying more personalized treatment strategies (10). A number of clinical trials using immune checkpoint inhibitors are underway and have shown limited utility for PD-L1 testing in identifying those most likely to respond.

\section{Malignant Mesothelioma in Situ}

Malignant mesothelioma in situ is a preinvasive lesion defined as a single layer of atypical mesothelial cells lining the pleural surface and characterized by loss of BAP1 and/or MTAP expression using immunohistochemistry. Criteria for diagnosis include recurrent pleural effusions, lack of pleural thickening and nodularity on chest imaging, and either no or only incidental findings at video-assisted thoracoscopic surgery (VATS) or thoracotomy. Given these criteria, diagnosis requires a multidisciplinary approach (40). Churg et al. showed that seven of ten patients with well-defined mesothelioma in situ developed MPM during a followup period of 12-92 months (40).

\section{Conclusions}

Diffuse MPM is a heterogeneous group of aggressive pleural tumors for which histological classification plays an increasingly important role in patient management and survival. Update of epithelioid mesothelioma includes pleomorphic subtypes, in which more than $10 \%$ of tumor cells show marked nuclear pleomorphism; this subtype is associated with the worst overall survival among epithelioid MPM. Considering that transitional mesothelioma is genetically closely related to sarcomatoid MPM, recent studies recommend it to be within a subgroup of sarcomatoid mesotheliomas. Stage and resectability remain key drivers of therapeutic strategies and outcomes. Given that none of the immunohistochemical markers has $100 \%$ specificity, an immunohistochemical panel should contain at least two mesothelial markers and two markers appropriate to the working diagnoses established based on routinely stained sections and any pertinent history including previ- ously diagnosed malignancies. There is an increasingly robust suite of diagnostic tools, including immunohistochemical stains for BAP1 and MTAP and $p 16$ FISH, for differentiating benign from malignant mesothelial proliferations in cytology and tissue specimens. Mesothelioma in situ has been recognized as a distinct clinicopathological entity for which more evidence is required to understand its natural history and treatment strategies that are proportional and targeted to the risk.

Conflict of Interest: The authors declare that they have no conflict of interest.

\section{References}

1. Churg A, Galateau-Salle F. The separation of benign and malignant mesothelial proliferations. Arch Pathol Lab Med. 2012;136(10):1217-26.

2. Finn RS, Brims FJH, Gandhi A, Olsen N, Musk AW, Maskell NA, et al. Postmortem findings of malignant pleural mesothelioma: a two-center study of 318 patients. Chest. 2012;142(5):1267-73.

3. Ogata S, Hiroi S, Tominaga A, Aida S, Kobayashi A, Tamura K, et al. Malignant pleural mesothelioma initially diagnosed on cervical lymph node biopsy. Pathol Int. 2009;59(8):592-4.

4. Henderson DW, Reid G, Kao SC, van Zandwijk N, Klebe $\mathrm{S}$. Challenges and controversies in the diagnosis of mesothelioma: Part 1. Cytology-only diagnosis, biopsies, immunohistochemistry, discrimination between mesothelioma and reactive mesothelial hyperplasia, and biomarkers. J Clin Pathol. 2013;66(10):847-53.

5. Michael CW, Bedrossian CC. The implementation of liquid-based cytology for lung and pleural-based diseases. Acta Cytol. 2014;58(6):563-73.

6. Hjerpe A, Ascoli V, Bedrossian CW, Boon ME, Creaney J, Davidson B, et al. Guidelines for the cytopathologic diagnosis of epithelioid and mixed-type malignant mesothelioma. Complementary statement from the International Mesothelioma Interest Group, also endorsed by the International Academy of Cytology and the Papanicolaou Society of Cytopathology. Acta Cytol. 2015;59(1):2-16.

7. Chirieac LR, Hung YP, Foo WC, Hofer MD, VanderLaan PA, Richards WG, et al. Diagnostic value of biopsy sampling in predicting histology in patients with diffuse malignant pleural mesothelioma. Cancer. 2019;125(23):4164-71.

8. Verma V, Ahern CA, Berlind CG, Lindsay WD, Shabason J, Sharma S, et al. Survival by Histologic Subtype of Malignant Pleural Mesothelioma and the Impact of Surgical Resection on Overall Survival. Clin Lung Cancer. 2018;19(6):e901-12. 
9. Marchevsky AM, Khoor A, Walts AE, Nicholson AG, Zhang YZ, Roggli V, et al. Localized malignant mesothelioma, an unusual and poorly characterized neoplasm of serosal origin: best current evidence from the literature and the International Mesothelioma Panel. Mod Pathol. 2020;33(2):281-96.

10. Nicholson AG, Sauter JL, Nowak AK, Kindler HL, Gill RR, Remy-Jardin M, et al. EURACAN/IASLC Proposals for Updating the Histologic Classification of Pleural Mesothelioma: Towards a More Multidisciplinary Approach. J Thorac Oncol. 2020;15(1):29-49.

11. Kadota K, Suzuki K, Sima CS, Rusch VW, Adusumilli PS, Travis WD. Pleomorphic epithelioid diffuse malignant pleural mesothelioma: a clinicopathological review and conceptual proposal to reclassify as biphasic or sarcomatoid mesothelioma. J Thorac Oncol. 2011;6(5):896-904.

12. Galetta D, Catino A, Misino A, Logroscino A, Fico M. Sarcomatoid mesothelioma: future advances in diagnosis, biomolecular assessment, and therapeutic options in a poor-outcome disease. Tumori. 2016;102(2):127-30.

13. Ismail-Khan R, Robinson LA, Williams CC Jr, Garrett CR, Bepler G, Simon GR. Malignant pleural mesothelioma: a comprehensive review. Cancer Control. 2006;13(4):255-63.

14. Klebe S, Brownlee NA, Mahar A, Burchette JL, Sporn TA, Vollmer RT, et al. Sarcomatoid mesothelioma: a clinical-pathologic correlation of 326 cases. Mod Pathol. 2010;23(3):470-9.

15. Mangano WE, Cagle PT, Churg A, Vollmer RT, Roggli VL. The diagnosis of desmoplastic malignant mesothelioma and its distinction from fibrous pleurisy: a histologic and immunohistochemical analysis of 31 cases including p53 immunostaining. Am J Clin Pathol. 1998;110(2):191-9.

16. Galateau-Salle F, Churg A, Roggli V, Travis WD, World Health Organization Committee for Tumors of the Pleura. The 2015 World Health Organization Classification of Tumors of the Pleura: Advances since the 2004 Classification. J Thorac Oncol. 2016;11(2):142-54.

17. Schulte JJ, Husain AN. Update on the pathologic diagnosis of malignant mesothelioma. Transl Lung Cancer Res. 2020;9(3):917-23.

18. Galateau Salle F, Le Stang N, Tirode F, Courtiol P, Nicholson AG, Tsao MS, et al. Comprehensive Molecular and Pathologic Evaluation of Transitional Mesothelioma Assisted by Deep Learning Approach: A Multi-Institutional Study of the International Mesothelioma Panel from the MESOPATH Reference Center. J Thorac Oncol. 2020;15(6):1037-53.

19. Dacic S, Le Stang N, Husain A, Weynand B, Beasley MB, Butnor $\mathrm{K}$, et al. Interobserver variation in the assessment of the sarcomatoid and transitional components in biphasic mesotheliomas. Mod Pathol. 2020;33(2):255-62.

20. Wu D, Hiroshima K, Yusa T, Ozaki D, Koh E, Sekine Y, et al. Usefulness of p16/CDKN2A fluorescence in situ hybridization and BAP1 immunohistochemistry for the diagnosis of biphasic mesothelioma. Ann Diagn Pathol. 2017;26:31-7.

21. Marchevsky AM, LeStang N, Hiroshima K, Pelosi G, Attanoos R, Churg A, et al. The differential diagnosis between pleural sarcomatoid mesothelioma and spindle cell/pleomorphic (sarcomatoid) carcinomas of the lung: evidencebased guidelines from the International Mesothelioma Panel and the MESOPATH National Reference Center. Hum Pathol. 2017;67:160-8.

22. Husain AN, Colby TV, Ordonez NG, Allen TC, Attanoos $\mathrm{RL}$, Beasley MB, et al. Guidelines for Pathologic Diagnosis of Malignant Mesothelioma 2017 Update of the Consensus Statement From the International Mesothelioma Interest Group. Arch Pathol Lab Med. 2018;142(1):89-108.

23. Ordonez NG. Application of immunohistochemistry in the diagnosis of epithelioid mesothelioma: a review and update. Hum Pathol. 2013;44(1):1-19.

24. Attanoos RL, Gibbs AR. 'Pseudomesotheliomatous' carcinomas of the pleura: a 10-year analysis of cases from the Environmental Lung Disease Research Group, Cardiff. Histopathology. 2003;43(5):444-52.

25. Davidson B, Totsch M, Wohlschlaeger J, Hager T, Pinamonti M. The diagnostic role of BAP1 in serous effusions. Hum Pathol. 2018;79:122-6.

26. Berg KB, Churg A. GATA3 Immunohistochemistry for Distinguishing Sarcomatoid and Desmoplastic Mesothelioma From Sarcomatoid Carcinoma of the Lung. Am J Surg Pathol. 2017;41(9):1221-5.

27. Churg A, Naso JR. The Separation of Benign and Malignant Mesothelial Proliferations: New Markers and How to Use Them. Am J Surg Pathol. 2020;44(11):e100-12.

28. Bott M, Brevet M, Taylor BS, Shimizu S, Ito T, Wang L, et al. The nuclear deubiquitinase BAP1 is commonly inactivated by somatic mutations and 3p21.1 losses in malignant pleural mesothelioma. Nat Genet. 2011;43(7):668-72.

29. Farzin M, Toon CW, Clarkson A, Sioson L, Watson N, Andrici J, et al. Loss of expression of BAP1 predicts longer survival in mesothelioma. Pathology. 2015;47(4):302-7.

30. Betti M, Casalone E, Ferrante D, Romanelli A, Grosso F, Guarrera S, et al. Inference on germline BAP1 mutations and asbestos exposure from the analysis of familial and sporadic mesothelioma in a high-risk area. Genes Chromosomes Cancer. 2015;54(1):51-62.

31. Cigognetti M, Lonardi S, Fisogni S, Balzarini P, Pellegrini V, Tironi A, et al. BAP1 (BRCA1-associated protein 1) is a highly specific marker for differentiating mesothelioma from reactive mesothelial proliferations. Mod Pathol. 2015;28(8):1043-57.

32. Weissferdt A, Kalhor N, Suster S. Malignant mesothelioma with prominent adenomatoid features: a clinicopathologic and immunohistochemical study of 10 cases. Ann Diagn Pathol. 2011;15(1):25-9.

33. Erber R, Warth A, Muley T, Hartmann A, Herpel E, Agaimy A. BAP1 Loss is a Useful Adjunct to Distinguish 
Malignant Mesothelioma Including the Adenomatoidlike Variant From Benign Adenomatoid Tumors. Appl Immunohistochem Mol Morphol. 2020;28(1):67-73.

34. Chapel DB, Husain AN, Krausz T. Immunohistochemical evaluation of nuclear 5-hydroxymethylcytosine (5-hmC) accurately distinguishes malignant pleural mesothelioma from benign mesothelial proliferations. Mod Pathol. 2019;32(3):376-86.

35. Illei PB, Rusch VW, Zakowski MF, Ladanyi M. Homozygous deletion of CDKN2A and codeletion of the methylthioadenosine phosphorylase gene in the majority of pleural mesotheliomas. Clin Cancer Res. 2003;9(6):210813.

36. Hwang HC, Pyott S, Rodriguez S, Cindric A, Carr A, Michelsen $\mathrm{C}$, et al. BAP1 Immunohistochemistry and p16 FISH in the Diagnosis of Sarcomatous and Desmoplastic Mesotheliomas. Am J Surg Pathol. 2016;40(5):714-8.
37. Hida T, Hamasaki M, Matsumoto S, Sato A, Tsujimura T, Kawahara K, et al. Immunohistochemical detection of MTAP and BAP1 protein loss for mesothelioma diagnosis: Comparison with 9p21 FISH and BAP1 immunohistochemistry. Lung Cancer. 2017;104:98-105.

38. Bruno R, Ali G, Giannini R, Proietti A, Lucchi M, Chella A, et al. Malignant pleural mesothelioma and mesothelial hyperplasia: A new molecular tool for the differential diagnosis. Oncotarget. 2017;8(2):2758-70.

39. Kinoshita Y, Hamasaki M, Yoshimura M, Matsumoto S, Iwasaki A, Nabeshima K. Hemizygous loss of NF2 detected by fluorescence in situ hybridization is useful for the diagnosis of malignant pleural mesothelioma. Mod Pathol. 2020;33(2):235-44.

40. Churg A, Galateau-Salle F, Roden AC, Attanoos R, von der Thusen JH, Tsao MS, et al. Malignant mesothelioma in situ: morphologic features and clinical outcome. Mod Pathol. 2020;33(2):297-302. 